\title{
Critical IIIness Polyneuromyopathy in a Patient with Disseminated Cryptococcal Infection
}

\author{
Jenn-Yu Wu, MD ${ }^{1}$ \\ Shang-Gin Wu, mo ${ }^{1}$ \\ Hsin-Yun Sun, MD ${ }^{2}$ \\ Ping-Hung Kuo, $\mathrm{MD}^{2}$ \\ Pan-Chyr Yang, MD, PhD ${ }^{2}$ \\ ${ }^{1}$ National Taiwan University Hospital Yun-Lin \\ Branch, Yun-Lin, Taiwan. \\ ${ }^{2}$ National Taiwan University Hospital, Taipei, \\ Taiwan.
}

\begin{abstract}
Critical illness polyneuromyopathy is frequent in patients in intensive care units. We report a case of critical illness polyneuromyopathy in a patient with disseminated cryptococcal infection in an intensive care unit. Journal of Hospital Medicine 2009;4:E3-E6. () 2009 Society of Hospital Medicine.
\end{abstract}

KEYWORDS: critical illness polyneuromyopathy, cryptococcus, insulin therapy.

ritical illness polyneuromyopathy (CIPNM) is frequent $\checkmark$ among critically-ill patients. ${ }^{1,2}$ CIPNM develops in criticallyill patients with systemic inflammatory response syndrome, sepsis, or multiple organ failure. We report a case of disseminated cryptococcal infection in a critically-ill patient who presented with treatment-refractory cellulitis. His recovery was complicated by difficulty in weaning from mechanical ventilation due to CIPNM.

\section{CASE REPORT}

A 49-year-old man developed skin swelling and tenderness over his right thigh 1 month prior to admission at a local hospital. He was a pigeon breeder by profession. His medical history was significant for hypertension and poorly controlled diabetes mellitus. His medications included prednisolone (15 mg per day), which he was taking for his chronic arthritis for about 6 months. He was admitted for treatment of cellulitis. Despite antibiotic treatment with ampicillin and sulbactam, his skin lesion became necrotic and gangrenous. Wound healing remained poor even after wound debridement. Patient developed dyspnea 2 weeks after admission and was transferred to the intensive care unit (ICU) of National Taiwan University Hospital for further management.

On transfer to ICU, he was drowsy and had an ear temperature of $37.1{ }^{\circ} \mathrm{C}$, pulse rate of 108 beats/minute, blood pressure of $132 / 82 \mathrm{~mm} \mathrm{Hg}$, and respiratory rate of 20 breaths/minutes. His pupils were isocoric with prompt light reflex. His right thigh wound was $6 \mathrm{~cm}$ in length with eschar formation and focal erythema. His total white blood cell count was $14.08 \times 10^{9}$ cells/L with $72.2 \%$ polymorphonuclear leukocytes. His blood glucose level was $326 \mathrm{mg} / \mathrm{dL}$. Other blood work, including hemoglobin, coagulation studies, and renal and liver function tests, were within normal range. Urinalysis was positive for glucose, but there was no hematuria, pyuria, or ketonuria. The chest radiograph (Figure 1) showed small nodules and peripheral infiltrates 


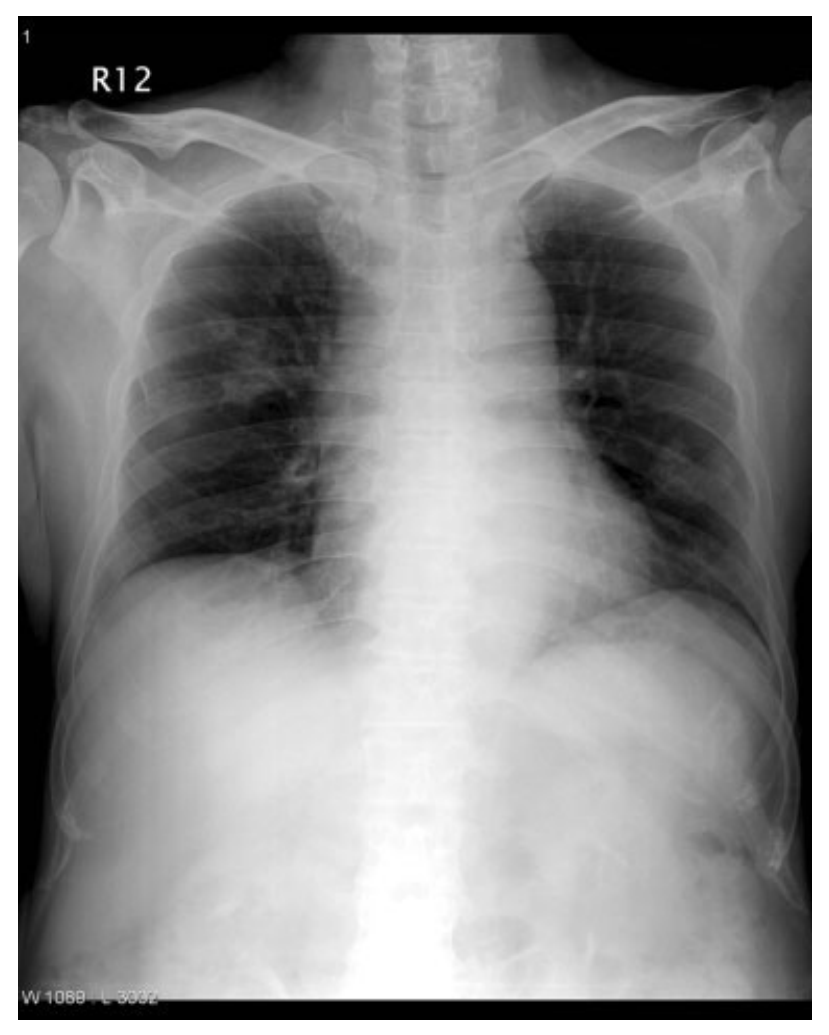

FIGURE 1. Chest radiograph on the admission day showing small nodules and peripheral infiltrates in bilateral lungs.

in both lung fields. Chest computerized tomogram (CT) scans found multiple cavitated lung nodules (Figure 2A,B). Human immunodeficiency virus (HIV) screening test was negative.

Soon after transfer to the ICU, the patient developed respiratory distress and became hemodynamically unstable. He was intubated and resuscitation efforts were started to stabilize him. Yeast-like organisms were found in the debrided tissue. CT-guided biopsy of the pulmonary nodules also found yeast-like organisms. Tissue cultures of skin wound and lung biopsy grew Cryptococcus neoformans. He was diagnosed with disseminated cryptococcal infection and Amphotericin B was started. His hemodynamic status stabilized 2 days after the start of Amphotericin B. Follow-up chest radiograph 1 week after starting Amphotericin B treatment showed resolution of pulmonary nodules. His right thigh skin lesion also healed gradually.

Unfortunately, he developed severe weakness of all extremities on the fourteenth ICU day, and had difficulty weaning from mechanical ventila-
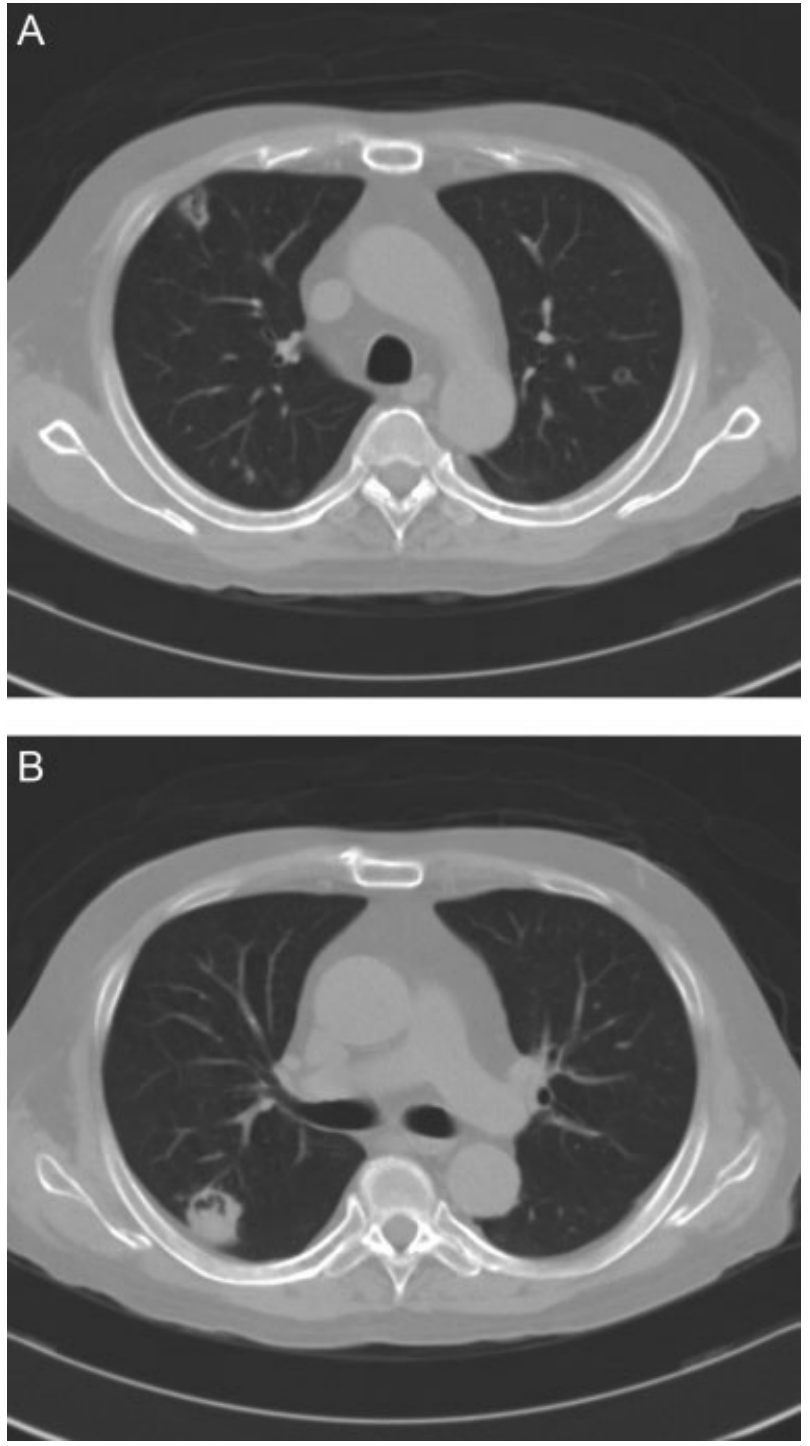

FIGURE 2. $(A, B)$ Chest computerized tomogram (CT) scans showing multiple cavitated nodules.

tion. Neurological examination found diffuse hyporeflexia. The nerve conduction velocity (NCV) study showed decreased amplitude in nerve conduction, with normal velocity. Electromyogram (EMG) found diffuse muscular fibrillation. Based on these findings, CIPNM was diagnosed. Patient underwent tracheostomy on the twenty-sixth ICU day due to the need for prolonged mechanical ventilation. His antifungal therapy was changed to intravenous fluconazole $600 \mathrm{mg}$ per day. Blood glucose levels were controlled with continuous insulin infusion and insulin dose was titrated to keep the blood glucose below 
$120 \mathrm{mg} / \mathrm{dL}$. He was finally weaned from mechanical ventilation on the forty-seventh day of ICU admission, and was transferred to the general ward. With physical therapy, he resumed his daily activities and was discharged from the hospital.

\section{DISCUSSION}

Cryptococcus rarely causes disseminated infection in healthy individuals. The vast majority of patients with cryptococcosis are immunocompromised due to conditions such as acquired immunodeficiency syndrome, prolonged treatment with corticosteroids, organ transplantation, malignancy, or diabetes mellitus. ${ }^{3,4}$ The patient in this report had 2 of the documented risk factors. In a study of 52 disseminated cryptococcosis patients, the most prevalent underlying condition was HIV infection (46\%). Out of these 52 patients, 28 were HIV-negative, and only 3 of them had no predisposing condition. ${ }^{4}$ Cutaneous cryptococcus infection could be a manifestation of disseminated cryptococcal infection, or may be a primary disease that would evolve into systemic disease several months later. ${ }^{5,6}$ The incidence of primary cutaneous cryptococcosis is much lower than systemic disease with cutaneous manifestation. Cellulitis, which is refractory to treatment with traditional antibacterial agents, should lead to consideration of fungal cellulites, such as cutaneous cryptococcosis or aspergillosis. Once cryptococcal cellulitis is diagnosed, other foci of cryptococcal infection should be sought.

CIPNM includes myopathies, acute neuropathies, neuromuscular transmission defects, or any combination of these disorders. ${ }^{2,7}$ CIPNM might arise in ICU patients who have systemic inflammatory response syndrome (SIRS), sepsis, or multiple organ failure. In patients with new onset limb weakness, disuse hyporeflexia, and difficulty in weaning from mechanical ventilation, CIPNM should be among the differential diagnoses. In 1 prospective trial, de Letter et al. $^{8}$ found CIPNM incidence of $33 \%$ in 98 ICU patients. A high APACHE III score, SIRS, and the use of aminoglycoside antibiotics are significant risk factors for the development of CIPNM. In various studies, the incidence of CIPNM ranges from $13 \%$ to $76 \%$. $^{2,7-10}$

The definitive diagnosis of CIPNM is established by EMG and NCV studies, which are characterized by (1) reduced amplitudes of compound muscles and sensory action potentials, (2) rela- tively normal conduction studies, and (3) widespread fibrillations and sharp waves. ${ }^{2}$ The mean mortality of patients who have developed CIPNM is $35 \%$, with a range from $0 \%$ to $73 \%$. About $45 \%$ of patients completely recover from CIPNM within 6 weeks to 12 months (with a mean duration of 4.5 months), with good supportive care, provided the underlying condition has been treated. ${ }^{2,9}$ There is little evidence to support a specific treatment modality for CIPNM. Mohr et al. ${ }^{1}$ reported that intravenous immunoglobulin may prevent or ameliorate CIPNM in the ICU, but more prospective and placebo-controlled studies are needed to confirm their result. In recent studies, ${ }^{11}$ strict blood glucose control with insulin was found to reduce the risk of CIPNM and duration of mechanical ventilation in both medical and surgical ICU patients.

In conclusion, CIPNM occurs frequently in ICU patients with SIRS, sepsis, or multiple organ failure, leading to prolonged ventilator dependence and increased morbidity and mortality. Risk factor prevention and intensive insulin treatment may reduce the incident of CIPNM and the duration of mechanical ventilation in ICU patients.

Address for correspondence and reprint requests: Ping-Hung Kuo, MD, Department of Internal Medicine, National Taiwan University Hospital, No. 7 Chung-Shan South Road, Taipei, Taiwan; Telephone: 886-2-23562905; Fax: 886-2-23582867; E-mail: kph712@ntuh.gov.tw

Received 18 December 2007; revision received 5 February 2008; accepted 9 February 2008.

\section{REFERENCES}

1. Mohr M, Englisch L, Roth A, Burchardi H, Zielmann S. Effects of early treatment with immunoglobulin on critical illness polyneuropathy following multiple organ failure and gram-negative sepsis. Intensive Care Med. 1997;23:11441149 .

2. Visser LH. Critical illness polyneuropathy and myopathy: clinical features, risk factors and prognosis. Eur J Neurol. 2006;13:1203-1212.

3. Diamond RD. Cryptococcus neoformans. In: Mandell GL, Bennett JE, Dolin R, eds. Principles and Practice of Infectious Diseases. Philadelphia: Churchill Livingstone; 2000: 2707-2718.

4. Jean SS, Fang CT, Shau WY, et al. Cryptococcameia: clinical features and prognostic factors. Q J Med. 2002;95: 511-518.

5. Bauza A, Redondo P, Rubio M. Primary cutaneous cryptococcal cellulitis secondary to insect bite in an immunosuppressed patient after liver transplantation. Clin Exp Dermatol. 2005;30:241-243.

6. Song IC, Hunter JG. Primary cutaneous cryptococcosis as the presenting manifestation of AIDS. Plast Reconstr Surg. 1992;90:1065-1067. 
7. Garnacho-Montero J, Amaya-Villar R, Garcia-Garmendia JL, Madrazo-Osuna J, Ortiz-Leyba C. Effect of critical illness polyneuropathy on the withdrawal from mechanical ventilation and the length of stay in septic patients. Crit Care Med. 2005;33:349-354.

8. De Letter MA, van Doorn PA, Savelkoul HF, et al. Critical illness polyneuropathy and myopathy (CIPNM): evidence for local immune activation by cytokine-expression in the muscle tissue. J Neuroimmunol. 2000;106:206-213.
9. Lorin S, Nierman DM. Critical illness neuromuscular abnormalities. Crit Care Clin. 2002;18:553-568.

10. Maher J, Rutledge F, Remtulla H, Parkes A, Bernardi L, Bolton CF. Neuromuscular disorders associated with failure to wean from the ventilator. Intensive Care Med. 1995;21:737-743.

11. Hermans G, Wilmer A, Meersseman W, et al. Impact of intensive insulin therapy on neuromuscular complications and ventilator-dependency in MICU. Am J Respir Crit Care Med. 2007;175:480-489. 